\title{
Asian-Canadian children and families involved in the child welfare system in Canada: A mixed methods study
}

\author{
Barbara Lee, Emse Fuller-Thomson, Barbara Fallon, Nico \\ Trocmé, and Tara Black
}

\author{
Version Publisher's PDF \\ Citation Lee, B., Fuller-Thomson, E., Fallon, B., Trocmé, N., \& Black, T. (2017). \\ (published version) Asian-Canadian children and families involved in the child welfare system in \\ Canada: A mixed methods study. Child Abuse \& Neglect, 70, 342-355. \\ doi:10.1016/j.chiabu.2017.06.022 \\ Copyright / License CC-BY-NC-ND \\ Publisher's Statement This is a Post-Print of an article published by Elsevier in Child Abuse and \\ Neglect in 2017. The published version is available at \\ https://doi.org/10.1016/j.chiabu.2017.06.022
}

How to cite TSpace items

Always cite the published version, so the author(s) will receive recognition through services that track citation counts, e.g. Scopus. If you need to cite the page number of the author manuscript from TSpace because you cannot access the published version, then cite the TSpace version in addition to the published version using the permanent URI (handle) found on the record page.

This article was made openly accessible by $U$ of $T$ Faculty. Please tell us how this access benefits you. Your story matters. 
Running head: ASIAN-CANADIAN CHILDREN AND FAMILIES

Asian-Canadian children and families involved in the child welfare system in Canada: A mixed methods study

Child Abuse and Neglect

2016

Barbara Lee ${ }^{1}$, Esme Fuller-Thomson ${ }^{2}$, Barbara Fallon ${ }^{2}$, Nico Trocmé ${ }^{3}$, and Tara Black ${ }^{2}$

Corresponding Author:

Barbara Lee

2080 West Mall

Vancouver, BC

Canada V6T $1 \mathrm{Z2}$

Telephone: (604) 822-9647

Fax: (604) 822-8656

E-mail: B.Lee@ubc.ca

1 School of Social Work, University of British Columbia, Vancouver, British Columbia, Canada

2 Factor-Inwentash Faculty of Social Work, University of Toronto, Toronto, Ontario, Canada

3 School of Social Work, McGill University, Montreal Quebec, Canada 


\section{Asian-Canadian children and families involved in the child welfare system in Canada: A mixed methods study}

\section{Introduction}

This study aims to understand the differences in child welfare involvement for AsianCanadian (East and Southeast Asian) versus White-Canadian children and families involved in the child welfare system in Canada, and to consider the implications and recommendations for service. This study began by replicating this author's original study, Author (2014), using the most recent 2008 Canadian Incidence Study of Reported Child Abuse and Neglect (CIS-2008). In the original study, author (2014) conducted secondary data analysis of the CIS-2003 and found a higher proportion of substantiated child maltreatment investigations with physical abuse as the primary maltreatment type for Asian-Canadian families, compared to non-Asian families. There were significantly more physical abuse investigations involving Asian-Canadian families that used "hit with object" as a form of physical maltreatment and "spanking" as a form of discipline, compared to physical abuse investigations involving non-Asian-Canadian families (Author, 2014, p. 543). Furthermore, the study found there was "an 11.25 times greater likelihood of child welfare placement for substantiated physical abuse investigations involving Asian families than those involving non-Asian families" (Author, 2014, p. 545). Ethnicity was the most significant predictor of out-of-home child welfare placement (Author, 2014). The consistent findings of disparate case characteristics and short-term service outcome between Asian-Canadian and White-Canadian children and families involved in the child welfare system using the CIS-2008 data, prompted a mixed methods research design. The current study examines child welfare workers and community service providers' interpretations regarding results from the secondary data analysis of the CIS-2008. 
Asian children and families are an under-studied population in child welfare research (Behl, Crouch, May, Valente, \& Conyngham, 2001). More attention to Asian children and families in the child welfare system is necessary. East and Southeast Asians combine to represent the fastest growing ethnic minority in Canada and the United States (Statistics Canada, 2010; U.S. Census Bureau, 2012). "According to the 2010 Census, the Asian population grew faster than any other race group in the United States between 2000 and 2010" (U.S. Census Bureau, 2012). Similarly in Canada, the proportion of Asian-born persons has steadily increased from 14\% in 1981 to $41 \%$ in 2006 (Statistics Canada, 2010). For the first time in history, the Asianborn proportion of the foreign-born population exceeded the European-born proportion in 2006 (Statistics Canada, 2010). Statistics Canada (2010) projects that the Chinese and Filipino population (currently the second and fourth largest visible minority group in Canada) will double in population in the next two decades. Furthermore, "the visible minority population would be over-represented in the younger age groups ... 36\% of the population under 15 years of age in 2031 would belong to a visible minority group, compared to $18 \%$ of persons aged 65 and over" (Statistics Canada, p. 1). The growing Asian visible minority population in Canada and United States prompts us to carefully consider the unique needs of these communities and how to best service the vulnerable children, youth, and families that come to the attention of the child welfare system.

There is no universal definition of child abuse and what constitutes violence against children varies substantially across the Southeast Asia region (Chan, Lam, \& Shae, 2011; Ju \& Lee, 2010; UNICEF, 2005). Many countries in Southeast Asia do not have established child welfare systems or legislation for the protection of children from maltreatment (UNICEF, 2005). Empirical studies suggest, however, that various forms of maltreatment occur in the population, 
and children could benefit from formal child welfare intervention in Southeast Asia (Chan et al., 2011; Ju \& Lee, 2011; Tang, 2006; Zhu \& Tang, 2012). Chinese adolescents in Hong Kong who experience physical abuse have high rates of health-compromising behaviors including sexual experimentation, non-fatal self-harm, and suicidal behaviors with low levels of social support (Tang, 2011). In many Southeast Asian cultures, personal or family shame, perceived disobedience, and fear of retribution prevent many cases of child abuse from being reported (Chan et al., 2011; Rhind, Leung, \& Choi, 1999; UNICEF, 2005; Zhu \& Tang, 2012). In Canada and the United States, where child welfare is an established system of protection, Asian populations are found to be under-represented in child welfare services, compared to all other ethno-racial groups (Dakil, Cox, Lin, \& Flores, 2011; Fluke et al., 2003; Hill, 2007). When involved in the child welfare system, Asian/Pacific Islanders have the highest proportions of reported physical abuse compared to all other ethno-racial groups in the United States (Dakil et al., 2011). Furthermore, Asian/Pacific Islanders were less likely to receive child protective service interventions such as family preservation, mental health, and substance abuse than other ethno-racial groups involved in the child welfare system for physical abuse in the United States (Dakil et al., 2011).

The Asian population has a diversity of needs. Huang, Calzada, Cheng, and Brotman (2012) found that immigrant Asian-American children in a community sample had higher levels of internalizing problems, lower levels of interpersonal relationship skills, and were less likely to be in good physical health compared to White children. Among the Asian sub-groups, Southeast Asian parents had the lowest average level of education and were more likely to live in poverty. Children of Southeast Asian immigrants were found to have the worst physical and mental health outcomes among the Asian sub-groups. Despite some evidence of need for services, Asian 
communities are under-represented in mental health (Leong \& Lau, 2001), physical health, and social services (Kim \& Keefe, 2010).

Behl et al. (2001) conducted a content analysis on the inclusion of ethnicity in child welfare research over 20 years and found that only $2 \%$ of the empirical literature in which ethnic breakdowns were reported, included Asian children and families. This lack of attention ignores the real and relevant issues in the Asian communities. The lack of child welfare research on Asian populations may contribute to the Asian "model minority" stereotype that claims that little or no government or social services are needed for this population (Kim \& Keefe, 2010; Leong \& Lau, 2001). The "model minority" stereotype reinforces the myth that an individual can overcome disadvantage and discrimination by simply working harder. This myth can negate the broader structural and systemic barriers that hinder access and equality. The harmful consequence is that Asian populations will continue to be made invisible in social service provision, practice standards, and research literature. This study aims to contribute to the knowledge-based regarding Asian-Canadian children and families involved in the child welfare system, and offer recommendations for working with this population.

\section{Literature Review}

There is a multitude of research regarding racial disproportionality and disparity (Ards, Chung, \& Myers, 1998; Fluke, Harden, Jenkins, \& Ruehrdanz, 2010; Hill, 2006; Hines, Lemon, Wyatt, \& Merdinger; 2004). However, diversity in ethno-racial affiliation and level of acculturation among racial groups are rarely considered. The consideration of cultural values and level of acculturation can provide insight into family functioning and how the child welfare system can respond to the family's needs. As such, a review of the literature was conducted to provide insight into Asian cultural values and belief systems regarding child-rearing, the effects 
of acculturation on child welfare involvement, and child welfare workers' perspectives on crosscultural practices. The research includes studies from the United States, Hong Kong, Korea, UK and Norway.

\section{Asian Cultural Values and Belief Systems Regarding Child-rearing}

Cultural values of filial piety, familism, and harmony are embraced by many Southeast Asian communities (Chao, 1994; Lau, 2010; Lieber, Fung, \& Leung, 2006; UNICEF, 2005; Zhai \& Gao, 2009). Filial piety and familism endorse hierarchical relationships, patriarchal authority, and family unity (Chao, 1994; Lau, 2010; Lieber et al., 2006; Zhai \& Gao, 2009). Traditionally, children across Southeast Asia are expected to be deferential to adults and to have subordinate status in the social hierarchy. Children are expected to not challenge or question adults as this is considered disrespectful and results in "loss of face" for the adult (Chao, 1994; Lau, 2010; Lieber et al., 2006; Zhai \& Gao, 2009). Generally, corporal punishment and physical sanctions by caregivers are an important aspect of child-rearing in many Southeast Asian cultures and considered "best practice" (Chao, 1994; Lieber et al., 2006; Tang, 2006; UNICEF, 2005). Violence in the home is considered a private family matter and it is perceived to be disrespectful to intervene in another family's privacy. Victims are further silenced as they are not expected to bring shame upon their family by disclosing concerns and "lose face" (Chan et al., 2011; Rhind et al., 1999). Maltreatment and outward aggression towards children is a sensitive topic that clashes with cultural traditions of peace and harmony, and therefore remains largely hidden across Asian communities.

\section{Effects of Acculturation on Child Welfare Involvement}

The Theoretical Framework of Child Maltreatment Among Asian Americans by Gao and Zhai (2009) offers a comprehensive explanatory framework to understand the specific cultural 
values and practices of Asian Americans, the influence of immigration and minority status, and the ways professionals interact with this population. The framework identifies factors that may contribute to the low probability of incidents, or the high probability of incidents, or the low probability of disclosure.

Asian cultural customs and child-rearing practices include folk remedy practice, filial piety and familism, and values of virginity. Filial piety and familism includes high expectation for children, the belief of physical punishment as an effective disciplinary strategy, parental authority and children's obedience, family harmony and stoicism, and family cohesion and mutual aid (Each of these characteristics are described in more detail in Gao and Zhai [2009]). These factors may increase or decrease the probability of child maltreatment incidents, as well as, decrease the probability of child maltreatment disclosure (Gao \& Zhai, 2009).

The immigration and minority status of Asian Americans include the migration experience and generational status in a family, minority status and discrimination in society, and the invisibility to the authorities (e.g., police, immigration officers). The immigration and acculturation experience can present multiple stressors, particularly for new immigrants and refugees, and across generations (e.g., parents versus children, or first, second, or third generation). This may lead to higher child maltreatment incidences (Gao \& Zhai, 2009).

The professional attitudes and practices of social service professionals include misunderstanding, discrimination and miscommunication, the foreign practice of out-of-home placement, professionals' understanding of clients from the same cultural background, and culturally sensitive practice (Gao \& Zhai, 2009). Professional unawareness and unfamiliarity of cultural characteristics of Asian communities could increase the probability of child maltreatment reports and responses among Asian Americans (Gao \& Zhai, 2009). On the other 
hand, "culturally sensitive approaches also tends to lead practitioners to ignore or tolerate abusive parenting and to rationalize child maltreatment as cultural customs or child discipline ... may lead to the low disclosure of incidence in this population" (Gao \& Zhai, 2009, p. 220).

The Culture and Child Maltreatment Decision-making Model by Terao, Borrego and Urquiza (2001) was developed to guide decision-making regarding cultural discipline practices and child maltreatment. The Culture and Child Maltreatment Decision-making Model (Terao et al., 2001) takes into account the parent's level of acculturation and the severity of the maltreatment incident, and thereby determines the intervention. This model proposes that child welfare clients "with a low level of acculturation should initially be offered psycho-educational services (e.g., teaching/informing parents about acceptable parenting practices as well as alternative models of discipline)" (p. 163). Parents with high level of acculturation should be provided therapeutic responses (e.g., psychotherapy) because parents with high acculturation are presumed to understand social sanctions against child maltreatment. The parents may have other environmental stressors (i.e., marital discord, parent-child relationship issues) or clinical issues (i.e., depression, substance abuse) each of these require intervention (Terao et al., 2001). The culture and child maltreatment decision-making model illustrates a bidirectional relationship between a psycho-educational and psychotherapeutic dynamic response based on the presenting concern.

Western approaches to "parent training" interventions have been aimed at reducing child conduct problems and risk of child maltreatment (Kim et al., 2008; Lau, Fung, Ho, Liu, \& Gudiño, 2011; Leung Tsang, Heung, \& Yiu, 2009). Parent training strategies encourage positive discipline methods such as praise and social reward to increase desired child behaviors and discourages physical discipline. Some studies have shown parent training to be efficacious in 
decreasing subsequent maltreatment with Chinese families in Hong Kong (Kim et al., 2008; Lau et al., 2011; Leung et al., 2009).

\section{Child Welfare Workers' Perspectives on Cross-cultural Practices}

The literature reveals distinct cultural values in child-rearing among Asian families and communities (Chao, 1994; Lau, 2010; Lieber et al., 2006; UNICEF, 2005). Studies involving the perspective of child welfare workers acknowledge the complexity of working across cultural differences (Chibnall et al., 2003; Clarke, 2011; Kriz \& Skivenes, 2012; Lee, Soeck, Djelaj, \& Agius, 2013). A transnational study on Norwegian and British child welfare workers found that communication challenges, differences in cultural values regarding child-rearing, service users' lack of understanding of the child welfare system and service users' lack of understanding of the role of social workers, contributed to uncertainty in assessing maltreatment among ethnic minority families (Kriz \& Skivenes, 2012). Standardized child protection assessment tools and actuarial risk assessments have been developed and implemented to minimize subjectivity and bias (Baird, Wagner, Healy, \& Johnson, 1999). However, there is still a great deal of latitude for worker professional judgment and discretion in case decisions (Chibnall et al., 2003; Lee et al., 2013; Rivaux et al., 2008). Scholars have written extensively regarding racial bias in child welfare decision-making (Hill, 2006; Fluke, 2011). Using case vignettes that include visual depictures of physical injuries, Jent et al. (2011) found that child welfare workers had high agreement upon what constitutes physical abuse versus normative child-rearing. The severity of inflicted injuries, the implement used, and the location of the injury were key factors that contributed to the determination of abuse.

There is limited empirical literature regarding Asian children and families involved in the child welfare system. The literature review revealed distinct cultural values and belief systems 
regarding familism and child-rearing in Asian communities. The Theoretical Framework of Child Maltreatment Among Asian Americans (Gao \& Zhai, 2009) and Culture and Child Maltreatment Decision-making Model (Terao et al., 2001) are two frameworks that inform the ways the child welfare system work with Asian communities. Studies involving the perspective of child welfare workers detailed the complexity of working across cultural differences (Chibnall et al., 2003; Kriz \& Skivenes, 2012; Lee et al., 2013).

\section{Methods}

This study aims to contribute to the knowledge-base of child maltreatment-related investigations involving Asian children and families. Using a mixed methods approach, this study highlights the similarities and differences in child welfare involvement for Asian-Canadian (East and Southeast Asian) versus White-Canadian children and families involved in the child welfare system in Canada, and considers the implications and recommendations for service.

\section{Research Questions}

This study uses an explanatory sequential mixed methods design to answer the following research questions:

1. What are the case characteristics and short-term service outcomes for Asian-Canadian children and families involved in the child welfare system?

2. What are child welfare workers and community service providers' experience and perception of working with Asian-Canadian children and families involved in the child welfare system?

\section{Research Design}

An explanatory sequential mixed methods approach "begins by conducting a quantitative phase and follows up on specific results with a second [qualitative] phase. The second phase is 
implemented for the purposes of explaining the initial results in more depth" (Creswell \& Clark, 2010, p. 81).

The first phase of this study began by conducting secondary data analysis of the 2008 Canadian Incidence Study of Reported Child Abuse and Neglect (CIS-2008) with a focus on Asian-Canadian children and families. A semi-structured interview guide was developed in consultation with a panel of child welfare experts to gather insight and interpretation of the CIS2008 results. A pilot test of the interview guide with a convenience sample of five child welfare researchers enhanced the rigor of the guide. The results from secondary data analysis of the CIS2008 were presented to focus group participants in a workshop, and the semi-structured interview guide elicited reflective insight on the complex issues facing Asian-Canadian children and families in the child welfare system.

\section{Secondary Data Analysis of the CIS-2008}

The CIS-2008 is the most comprehensive and representative study of the incidence and characteristics of reported child maltreatment-related investigations in Canada (Trocmé et al., 2010). A detailed overview of the methodology used in the CIS-2008 can be found in Trocmé et al. (2010). The current sample from the CIS-2008 included child maltreatment-related investigations involving Asian-Canadian and White-Canadian caregivers and children 15 years of age and under at the time of case opening. The unit of analysis was child-maltreatment-related investigations at the family level. Ethnicity in the CIS-2008 is determined at the caregiver level. The CIS-2008 collects caregiver ethnicity based on the 1996 Census categorizations. Information regarding immigration status, level of acculturation and affiliation is not available in the CIS2008 data. The determinant of Asian-Canadian was the combination of "Chinese" and "Southeast Asian other than Chinese" backgrounds such as Vietnamese, Korean, Japanese, 
Filipino, Cambodian, Laotian, Hmong, and Samoan. The case was defined as Asian-Canadian in cases of child welfare involvement in which at least one caregiver was Asian-Canadian (including a second caregiver who was White-Canadian, excluding a second caregiver who was Aboriginal or Black). Where all caregivers were White-Canadians, the case was defined as White-Canadian. The sample was comprised of an unweighted sample of 10,770 child maltreatment-related investigations involving 380 Asian-Canadian and 10,390 White-Canadian children and families.

Two sets of weights were applied to the unweighted sample: an annualization weight and a regionalization weight. The annualization weight was used to derive an annual estimate of investigated children. Regionalization weight was used to derive representative estimates for all of Canada. A detailed overview of the CIS-2008 weights can be found in Trocmé et al. (2010).

In this sample, there were a lower proportion of child maltreatment-related investigations involving Asian-Canadian than White-Canadian children and families (unweighted sample of 380 vs. 10,$390 ; 3.5 \%$ vs. $96.5 \%$, respectively). Without adjustments, child maltreatment investigations and substantiated investigation results may be skewed by the differences in proportion of the ethno-racial groups. An adjusted means weight was used to correct the proportional imbalance between the ethno-racial groups. The adjusted means weight preserve the sample size for Asian-Canadian and White-Canadian children and families involved in child maltreatment-related investigations while generating the same proportional distribution as the full weights. The adjusted means weight compensates for the differences to determine the proportion of child maltreatment investigations and substantiated investigation if there were no differences between the ethno-racial groups.

Pearson's chi-square $\left(\chi^{2}\right)$ was used for all bivariate analyses in SPSS, version 22. The 
Bonferroni method was used to control the overall false positives (Type I error) when multiple comparisons were conducted (Bland, 1995). Statistical significance was identified at $p<.001$. The case characteristics examined in the study include referral source, investigation type, and primary maltreatment type. The short-term service outcome examined in the study include substantiation decision and decision to transfer to ongoing child protection services.

\section{Focus Groups}

This study used four focus groups with child welfare workers at two purposive sampled child welfare agencies and one separate round-table discussion with community service providers from a convenience sample of multicultural family service agencies. The community service providers were included in the sample to determine whether there are other considerations that need to be explored outside of the child welfare system. Upon preliminary analysis, it was determined the responses of the child welfare workers and the community service providers were not substantively different, and therefore all participant responses were retained for further analysis.

Child welfare workers were recruited via email from the child welfare agency staff listserv and poster advertisements were displayed in common areas in the two local child welfare agencies. The community service providers were recruited via email through this researcher's academic, professional, and personal networks, as well as poster advertisements at social service agencies. Participants consented to voluntarily partake in the focus groups and did not receive any monetary incentive for their involvement. The demographic, education, and practice experience of the focus group participants are presented in Table 1.

The total focus group sample was comprised of 18 ethnically diverse child welfare workers and community service providers. The focus groups were composed of 15 child welfare 
workers, and 3 community service providers. The focus group participants were predominantly female $(n=15)$, Asian $(n=10)$ or Black $(n=4)$, and the majority had a graduate degree $(n=11)$. Half the participants had 10 or more years of direct child welfare experience in multiple positions including intake screening/investigations, ongoing family services, or children's services.

Each focus group with child welfare workers was held in a private room at a local child welfare agency. The roundtable discussion with community service providers was held at a local community agency. The focus groups lasted on average 1.5 hours. The semi-structured interview with child welfare workers and community service providers included questions regarding the differences between Asian and non-Asian children and families involved in the child welfare system, the considerations in determining service provisions for Asian children and families involved in the child welfare system, and impressions and interpretations of the quantitative descriptive findings from the CIS-2008. The focus group discussions were audio-recorded and transcribed verbatim. At all phases of the study, this researcher engaged in memo writing, field notes, and process journals to assist with reflexivity, to uncover hidden assumptions and bracket prior knowledge (Hennink, 2007). Additional feedback and content validity were gathered through a follow-up community presentation and consultation with 13 child welfare workers and community service providers. The focus groups were conducted by the same facilitator (Author) to assist with maintaining consistency and allowed more carryover from one interview to the next for a more "organic" understanding of the issues (Brod, Tesler, \& Christensen, 2009).

Data analysis of the focus group responses was conducted through a series of iterative phases by hand and in NVivo, version 10. The first phase of analysis involved holistic coding by hand. Holistic coding is a "preparatory approach to a unit of analysis of data before a more 
detailed coding or categorization process" (Saldana, 2013, p. 142). The transcripts were read in their entirety to gain familiarity with the information within the context of the focus group discussion. The data were segmented into smaller manageable parts for the identification of themes that emerge from the focus group discussion (Hennink, 2007). A constant comparative method was used to gain a thorough understanding of the various views in the discussion (Boeije, 2002). This method compares the preliminary themes identified within one focus group discussion, as well as between focus group discussions, to determine whether the same ideas appeared or whether the preliminary themes need to be further refined (Boeije, 2002).

Following this initial phase, transcripts were uploaded into NVivo, version 10 for further analysis. A second cycle of coding was conducted using structural and descriptive coding (Saldana, 2013). Structural coding is a "question-based coding" that is most suitable for research with multiple participants, standardized or semi-structured data-gathering protocols, hypothesis testing, or exploratory investigations (Saldana, 2013, p. 84). The data was segmented based on the questions and responses as outlined in the semi-structured interview guide to identify major categories or themes. Descriptive coding documents the broader topic of discussion by categorizing the data together to provide a complete summary of the coded themes. "It is essential groundwork for second cycle coding and further analysis and interpretation" (Saldana, 2013, p. 89). Child welfare workers and community service providers were consulted on the identified preliminary themes, which were agreed upon by consensus (Elliott $\&$ Timulak, 2007).

Finally, thematic analysis was conducted to identify common and important issues within the data (Boyatzis, 1998). The synthesis of findings was verified through a final feedback group comprised of child welfare workers and community service providers to ensure the interpretation 
of the data was accurate (Elliott \& Timulak, 2007). The focus group findings were integrated with the CIS-2008 findings for an overall mixed method results and discussion.

\section{Results}

\section{CIS-2008 Findings}

The demographic characteristics of child maltreatment-related investigations involving Asian-Canadian and White-Canadian children and families are presented in Table 2. AsianCanadian households, compared to White-Canadian households, were more likely to consist of two caregivers ( $81.3 \%$ vs. $59.2 \%)$, both caregivers were more likely to be biological (93.8\% vs. $63.5 \%$ ), and employed full-time ( $73.4 \%$ vs. $56.3 \%$ ). Gender of the investigated child, age of the investigated child, and number of children in the family were comparable. The case characteristics of child maltreatment-related investigations, whether or not substantiated, are presented in Table 3.

Type of child maltreatment-related investigation. The type of child maltreatmentrelated investigations included "risk investigation only" or "maltreatment investigation". "Risk investigation only" involves a risk of future maltreatment but no specific incident or allegation of maltreatment (e.g., parent's drinking places child at risk for physical abuse or neglect, but no specific allegations or incident is suspected; Trocmé et al., 2010). A maltreatment investigation pertains to investigations involving a specific incident or event of maltreatment (e.g., a child was allegedly physically abused). In the CIS-2008, Asian-Canadian children and families had a higher proportion of maltreatment investigations compared to White-Canadian children and families $(84.8 \%$ vs. $74.3 \%, \mathrm{p}<.001)$.

Primary maltreatment type. In the CIS-2008, child maltreatment-related investigations involving Asian-Canadian children and families for physical abuse was almost twice that of 
White-Canadian children and families involved in the child welfare system (35.5\% vs. $18.9 \%, \mathrm{p}$ $<.001)$. On the other hand, the proportion of neglect, emotional maltreatment, and exposure to domestic violence was similar for child maltreatment-related investigations involving AsianCanadian and White-Canadian children and families.

Substantiation of child maltreatment concerns. Substantiated cases of maltreatment are when the balance of evidence indicates that abuse or neglect has occurred. Suspected is when there is not enough evidence to substantiate maltreatment, but no certainty that maltreatment can be ruled out. Unfounded is when the balance of evidence indicates that maltreatment has not occurred (Trocmé et al., 2010). In the CIS-2008, a slightly higher proportion of child maltreatment investigations involving Asian-Canadian children and families were substantiated than White-Canadian children and families (55.6\% vs. $46.5 \%)$. Therefore, further analyses were conducted to examine substantiated child maltreatment investigations involving Asian-Canadian and White-Canadian children and families in the child welfare system. The findings are presented in Table 4.

Referral sources. A higher proportion of reports for substantiated child maltreatment investigations involving Asian-Canadian children and families were from professional referral sources in comparison to White-Canadian children and families involved in the child welfare system $(90.2 \%$ vs. $75.6 \%, \mathrm{p}<.001)$. While the referral source for White-Canadian children and families were primarily from professional sources, White-Canadian children were referred more than twice that of Asian-Canadian children and families from non-professional sources such as self-referrals, family, friends, neighbours, and anonymous/other sources.

Primary maltreatment type for substantiated child maltreatment investigations. Child maltreatment investigations involving Asian-Canadian children and families were 
substantiated primarily for physical abuse (36.6\%), followed by exposure to intimate partner violence (31.2\%), neglect (22.6\%), emotional abuse (8.8\%), and sexual abuse (estimates too small to report according to CIS regulations) in the child welfare system. In contrast, child maltreatment investigations involving White-Canadian children and families were substantiated primarily for exposure to intimate partner violence (34.0\%), followed by neglect (31.3\%), physical abuse (21.1\%), emotional maltreatment (9.9\%), and sexual abuse (estimates too small to report according to CIS regulations) in the child welfare system.

Transfer to ongoing child protection services. Transfer to ongoing child protection service is the practice decision to transfer a family to receive ongoing child protection services for continued support and family services. In the CIS-2008, substantiated child maltreatment investigations involving Asian-Canadian children and families were less than half that of WhiteCanadian children and families to be transferred to ongoing child protection service (19.3\% vs. $43.1 \%, \mathrm{p}<.001)$.

\section{Focus Group Findings}

Type of child maltreatment-related investigation. Focus group participants suggested the maltreatment-related investigations involving Asian children and families were typically due to physical discipline, but were likely low severity and single incidences of involvement with the child welfare system. One worker indicated the closed and rigid family boundaries among AsianCanadian families contribute to the type of child maltreatment-related investigations: “...quiet family and children are not encouraged to speak about private family matters so then the only time we [the child welfare system] do get involved is when an incident has already happened. So we [the child welfare system] are not getting in at the early help stages." 
Participants believe that the use of physical discipline is considered a "cultural norm" in the Asian-Canadian families they investigated. The child welfare workers report that often their role involved psycho-education interventions to educate parents: "We talked to parent about, it [physical discipline] is not appropriate, there are other ways of discipline and giving them some strategies, we sometimes never saw the family again.” One participant further explained: "It's different and they [Asian-Canadian families] don't understand this formal system ... if you want to engage with a family you do have to be flexible and open in a culturally respectful way that is very different than the professionalism of Western notions of how you work with a family within the context of child protection."

Primary maltreatment type. Focus group participants discussed and differentiated physical discipline from physical abuse. One participant suggests: "Discipline and corporal punishment is not necessarily equivalent to physical abuse." Another participant further elaborated: "It is a form of teaching, but it's not like abuse. Abuse is when they [caregivers] just hit for whatever reason. They [abusive caregivers] just hit out of anger." Another participant referenced Section 43 of the Canadian Criminal Code which permits the use of physical force and outlines the boundaries of such practice: "Although the law allows a certain type of corporal punishment, there is a line that you cannot cross, in terms of age or where you hit, how you hit." In regard to the low proportion of investigations involving Asian-Canadian families for sexual abuse, one participant suggests that child welfare workers "have the myth that it [sexual abuse] would not happen often because the [Asian] culture is quite traditional. In reality, sometimes [the sexual abuse is] more, and more serious because they [the child victim] did not disclose. So that's why, when we [child welfare workers] do intake [assessment] with Asian families, we have to pay more attention to this." 
Substantiation of child maltreatment concerns. Focus group participants indicated that language barriers may impede investigated Asian-Canadian caregivers full participation in the child welfare decision-making process: "When you [Asian-Canadian caregiver] cannot explain yourself and then you get even more nervous ...you're talking, you're not understand[ing], you're not listening."

Referral sources. Focus group participants discussed the dynamics of Asian-Canadian families as inhibitors for non-professional referral sources. Several participants indicated that “the Asian culture is a more 'close-knit group'.” Another participant reported: "I hear a lot of times people saying: 'I don't want to create trouble for other people's families' ... There is maybe a lot more reservation in some Asian populations about opening, getting involved in other families' business." Participants acknowledged that professional referral sources have a professional obligation to contact child welfare services for any maltreatment, regardless of whether it was in the context of physical discipline. One participant suggests: "Maybe when the lady is going to the doctor reporting something, the doctor has an obligation to call us. That's the way we [the child welfare system] come to know. The child is going to school with a mark for whatever, they [the school] just call us." Another participant elaborated: "The teacher has no context within what type of discipline was used and so you cannot guarantee that CAS will not come to your door. So I always tell them [caregivers], if you don't want CAS at your door, refrain [from any hitting]."

\section{Primary maltreatment type for substantiated child maltreatment investigations. In} regard to the higher proportion of substantiated investigations involving Asian-Canadians for physical abuse, participants repeated that this was not physical abuse but rather physical discipline. The notion of culturally sanctioned forms of discipline was discussed: "I think use of 
corporal punishment is quite common in Asian populations. It's quite the norm." One participant indicated their child welfare response was determined by the caregiver's familiarity with the Canadian laws and their level of acculturation: "If a family came [to Canada] just last year or this year and they use physical discipline, I try to educate them. But the other one [not a new immigrant], I hold them responsible. I tell them [the caregiver], you are here, you learnt everything, you knew the system, and you are still using it [physical discipline], no, not acceptable."

Participants discussed the broader structural issues related to poverty that may lead to a judgment of neglect for Asian-Canadians: "Neglect, very often, is because it's a conflict between the institution and the way that the parents are dealing with the situation. They don't have a doctor, they don't bring their lunch, other issues like that, are called in by these professionals and the educations. That we identify as neglect." One participant discussed a case in which the caregivers thought they were providing care and basic needs for their family by agreeing to have a marijuana grow-op in the home; however, the child welfare system deemed their source of income as child maltreatment and apprehended the children:

I spoke to a mother who said, I had no idea that the children would be an issue because if somebody had told me that and said to me, I'm going to set you up in a grow-op house but if you're caught your children might be taken away, a lot of families wouldn't do it. But they had no clue that that was one of the effects of getting involved in it, they heard money and they heard, we'll protect you.

Ongoing child protection services. Focus group participants indicated a number of possible reasons for the lower proportion of substantiated child maltreatment investigations involving Asian-Canadian children and families that were transferred to ongoing child protection service. The reasons included the perceived lower severity of child maltreatment and single incident of child maltreatment, family refusal of continued child welfare service, and child 
welfare workers' lack of knowledge regarding the family needs. One focus group participant indicated: "We're not transferring them [the family] to ongoing service because we don't have a full depth and breadth of understanding of the family. To me, that's about language barriers and cultural barriers."

One participant indicated that a child welfare intervention may silence a family and further inhibit disclosure or discussion: "We have an intervention and people [child welfare workers] are going to come into our [family] home and ask us whether this [physical discipline] is used anymore. You're [family is] not allowed to talk about this anymore and there's a silencing that is occurring. I don't know whether real intervention has occurred in terms of changing and achieving some broader considerations for child-rearing." The participant further suggests that continued child welfare services may be needed for genuine change to occur:

Some of the families I've worked with around things like physical discipline, even the parents themselves, two parents, can have very different views. One parent can be completely on the opposite end of the spectrum, and it takes a lot of work and engagement to even get to that place of being able to understand where each other is coming from. Being able to do that in one visit in saying no, this physical discipline is not appropriate, I can't see that just changing someone's mind completely in terms of their views of physical discipline and why they use it. I'd be very skeptical of whether you could do that work in one visit.

The focus group participants discussed the lack of early intervention and supports for families, as well as the lack of culturally appropriate services for families. However, continued child welfare service is often voluntary and families must see the need: "we try to connect them to resources but if the families don't see the importance ... it seems more as an imposition."

\section{Discussion}

The CIS-2008 findings provide a descriptive overview of Asian-Canadian children and families involved in the child welfare system. When these findings were presented to several focus groups of child welfare workers and community service providers, practice insights and 
context for the CIS-2008 data were elucidated. Several overarching themes were noted regarding Asian-Canadian children and families and their involvement in the child welfare system: "closeknit" family dynamics inhibiting disclosure and child welfare involvement, culturally normative disciplinary practices are defined in the Canadian context as "abuse", lack of mutual understanding between child welfare professionals and investigated Asian-Canadian families, and child welfare services should be based on the client's need.

\section{“Close-knit” Family Dynamics Inhibiting Disclosure and Child Welfare Involvement}

Focus group participants described Asian-Canadian families as having "close-knit," "private," reserved and "rigid" structures, and these dynamics have been described as inhibiting disclosure and child welfare involvement. Participants indicated that the value of family privacy prevents disclosure of child maltreatment concerns, and professional are usually only involved once an incident has already occurred and have been reported to the child welfare system. This emphasis of family privacy also discourages individuals from self-reporting and prevents nonprofessionals from intervening in other families' concerns and contacting child welfare on their behalf. This is in line with the Theoretical Framework of Child Maltreatment Among Asian Americans (Zhai \& Goa, 2009) and the literature that acknowledges that the breach of family privacy is considered disrespectful behavior in Asian cultures because it brings shame to the family (Rhind et al., 1999; UNICEF, 2005; Zhai \& Goa, 2009).

If the child welfare system is involved with the family, the closed and rigid boundary of the family system keeps professional service providers at the periphery and prevents further involvement. The value of family privacy places a limit on how much information is provided to child welfare workers by the family member. On the other hand, the family's desire for privacy is used by child welfare workers to leverage change because parents are willing to no longer use 
physical discipline in order to prevent continued or future child welfare involvement. While focus group participants acknowledge that child welfare involvement with Asian-Canadian families may provide the necessary psycho-educational intervention as proposed in the Culture and Child Maltreatment Decision-making Model (Terao et al., 2001), it may also silence and inhibit disclosure or discussion as a way to maintain the "close-knit" boundary of the family system. Given the private nature of Asian-Canadian families, participants reported the need for greater attention, care and cultural sensitivity in understanding the family concerns, particularly around taboo topics such as sexuality, abuse, and domestic violence.

\section{Culturally Normative Disciplinary Practices are Defined in the Canadian Context as "Abuse"}

The Canadian Criminal Code Section 43 parameters of "reasonable force", child welfare definitions of abuse, and culturally sanctioned methods of discipline collide in the discussion of Asian-Canadian involvement in the child welfare system. Section 43 of the Canadian Criminal Code states: "Every school teacher, parent or person standing in the place of a parent is justified in using force by way of correction toward a pupil or child, as the case may be, who is under his care, if the force does not exceed what is reasonable under the circumstance" (Barnett, 2008, p. 2). The permission to use "reasonable force" stands in opposition to child welfare's role in ensuring the safety and protection of children from maltreatment (Barnett, 2008). There have been proposals for the reform and repeal of the Criminal Code, Section 43 (e.g., Canadian Foundation for Children, Youth and the Law v. Canada [Attorney General]; Durrant, Trocmé, Fallon, Milne, \& Black, 2009,) but without success. Asian-Canadian families are in the crossroads between what are legally permissible disciplinary methods in Canada and the child welfare definition of physical abuse. Author (2014) found that $75.6 \%$ of substantiated physical abuse investigations involving Asians had noted "spanking as a form of discipline." 
Focus group participants in this study identified this tension and contradiction in their involvement with families. Participants acknowledge professionals have a "duty to report" and without knowing whether the use of force was in the context of discipline, the discovery of a mark on a child or disclosure of the use of physical force on a child may create concerns of physical maltreatment. Participants confirm that the use of physical force on a child can be categorized as physical abuse based on the Ontario Child Welfare Eligibility Spectrum (OACAS, 2006) and may warrant eligibility for child protection involvement according to the Child and Family Service Act section 37(2). Participants indicate that upon investigation of alleged child maltreatment, the confirmation or admission of physical force used on the child can result in a substantiated child maltreatment investigation outcome. Nevertheless, participants acknowledge that this may not be physical abuse but rather culturally normative discipline practices by way of corporal punishment.

Many studies have examined the use of physical discipline in Asian families and confirm the prevalence of such practices across the East and Southeast Asia regions (Chao, 1994; Ho et al., 2012; Lau, 2010; Park, 2001; Tang, 2006). The use of corporal punishment among Chinese parents has been described as teaching and training children to be deferent and respectful to elders; motivating academic, social, and moral character; instilling strong masculine character among boys; and training children to be able to endure hardship (Chao, 1994; Lieber et al., 2006; Tang, 2006; Zhu \& Tang, 2012). However, parental intentions may not be understood or accepted by the children experiencing corporal punishment (Chan et al., 2011; Lau, 2010; Zhu \& Tang, 2012). Zhu and Tang (2012) found that although Chinese children perceived their parent's use of physical force as "loving punishment," the children strongly stated that such harsh discipline should be replaced by more respectful, non-violent, solution-focused disciplinary 
methods. To negotiate this complex tension between the Canadian Criminal Code, Section 43 parameters of "reasonable force," child welfare definitions of abuse, and culturally sanctioned methods of discipline, child welfare workers in this study reported utilizing a critical reflexive approach in assessing child maltreatment. In their practice, child welfare workers examine the broader historical, social-economic, and cultural context of child-rearing as viewed through the lens of the Ontario provincial child welfare system. Participants acknowledge that ultimately the level of severity, patterns of harm and multiplicity of complex concerns is what determines the need for child welfare intervention. Participants indicated the need for a delicate balance of recognizing cultural normative discipline methods, but also when such practices breach acceptability and may be considered abusive.

\section{Lack of Mutual Understanding Between Child Welfare Professionals and Investigated Asian-Canadian Families}

Many countries in Southeast Asia do not have established child welfare systems or legislation for the protection of children from maltreatment (UNICEF, 2005). Focus group participants in this study acknowledge that Asian-Canadian families may not be familiar with the role of social workers or the child welfare system. Similar to Kriz and Skivenes (2012), participants in this study noted that child welfare workers might be equated with police or other authorities that instill fear and unquestioned compliance in cooperation. Child welfare workers also do not have a good understanding of Asian-Canadian families due to language and cultural barriers, and the "close-knit" private family dynamics. This lack of understanding on both sides may result in missed opportunities for early intervention, and for meeting the needs of the child and family. Dakil et al. (2011) found the detection of alleged concerns for physical abuse among Asian Americans often does not translate to continued ongoing services or referral to community resources. 


\section{Child Welfare Services Should be Based on Clients' Needs}

Based on the different patterns of involvement between Asian-Canadian and WhiteCanadian children and families in the child welfare system, focus group participants in this study suggested a number of "best practice" principles for working cross-culturally. Participants suggest engaging with families in more "flexible," "open," and "culturally respectful way," which means doing things creatively and differently with members of diverse cultural communities. This may involve respecting the family's views of whether or not to use a translator, conducting additional home visits to build rapport and engaging in more in-depth conversations, knowledge sharing and conversations about what child welfare and optimal child well-being means, equipping caregivers with a range of parenting strategies and community resources to best care for their children, and operating from a strengths-based perspective that includes respecting the client's determination of service need. These suggestions address some of the barriers to access health services (Kim \& Keefe, 2010) and mental health service (Garland et al., 2003; Leong \& Lau, 2001) that has been identified among Asian populations.

\section{Limitations}

It is important to acknowledge that the Asian ethno-racial category encompasses many linguistic, cultural, traditional, spiritual, and socio-economical-political differences; however, more discrete categorization of Asian heritage including immigration status was not available in the CIS-2008 dataset. In studies of ethnicity, Breton (2005) found that patterns of ethnic retention and incorporation vary among ethnic groups, and do not evolve in the same way across generations. Thus, it is important to differentiate specific ethnic groups and their immigration status, rather than generalizing attributes to an ethnic group as a whole. Caution needs to be considered in interpreting the results of the study. Also, ethno-racial identity was determined at 
the caregiver level rather than the child level and it was measured by the judgment of the child welfare worker responsible for the investigation.

The CIS-2008 examines child welfare investigations and therefore, is unable to account for incidents of child maltreatment that were not reported to child welfare authorities, screened out and not investigated, and new reports for already opened child welfare cases.

Focus group participants were drawn from an urban area in southern Ontario, Canada. Therefore, the child welfare practices and perspectives may not be generalizable to other provinces or contexts. Missing are the voices and perspectives of the child welfare service users, as well as other professionals providing service to Asian-Canadian families involved in the child welfare system.

\section{Implications of Findings and Recommendations}

\section{Evidence-informed practices are needed to guide intervention with Asian-Canadian} children and families. Understanding the profile of child welfare investigations involving Asian-Canadian children and families is helpful to identify concerns that may warrant preventative intervention before child welfare involvement. Further evaluative research on Asian-Canadian children and families involved in the child welfare system can provide insight into what is currently working well and to identify areas for improvement.

2. Discrete categories of Asian populations need to be available for research. In the field of child welfare where little research has been conducted for Asian children and families (Behl et al., 2001), the mere existence and availability of Asian categorization can provide a wealth of knowledge for research and discovery. However, as proposed in the Theoretical Framework of Child Maltreatment Among Asian Americans and the Culture and Child Maltreatment Decision-making Model, the specific cultural values and practices of Asian 
Americans, the influence of immigration and minority status, and level of acculturation needs to be carefully considered in working with Asian populations. This information needs to be available for research and further analysis. The availability of data regarding ethnicity, race, and immigration status may offer a more accurate profile and understanding of the issue.

\section{Community engagement and outreach with Asian-Canadian communities are}

recommended. The focus group raised awareness that Asian-Canadian families and communities' lacked awareness and understanding of the child welfare system. This lack of mutual understanding may result in missed opportunities for early intervention and for meeting the needs of Asian-Canadian children and families. In attempts to gain mutual understanding and collaboration, community engagement and outreach from child welfare and Asian-Canadian communities can help forge positive relationships. Furthermore, protocols and collaborations between child welfare and ethno-specific agencies can provide joint support to Asian-Canadian children and families.

\section{There needs to be cross-cultural training for child welfare workers to promote cultural} sensitivity and awareness in child welfare organizations. Most child protection workers in Canada were White/Caucasian (Fallon, MacLaurin, Trocmé, \& Felstiner, 2003) and may not be familiar with the distinct customs and cultures in Asian populations. Some of the specialized knowledge regarding Asian-Canadian children and families include an understanding of cultural child-rearing practices such as the value and purpose of corporal punishment, distinct cultural values and belief systems regarding filial piety and familism, ethnic affiliation and level of acculturation, awareness of acculturative stress, and the practice of traditional healing methods.

\section{Conclusion}


Asian children and families are an under-represented (Dakil et al., 2011; Fluke et al., 2011; Hill, 2007) and under-studied population in child welfare research in Canada (except Author, 2014). The social service sector needs to be equipped and guided by an evidence-based approach to meet the needs of diverse populations. This study explored the demographic and case characteristics, short-term service outcome, and child protection workers' experiences and perceptions of working with Asian-Canadian children and families involved in the child welfare system.

This study used a mixed method approach to build a comprehensive understanding of Asian-Canadian children and families involved in the child welfare system at national and local levels. The results from secondary data analysis using the CIS-2008 indicated substantive differences between Asian-Canadian and White-Canadian children and families investigated by child welfare agencies. Differences were found with respect to the household composition, investigation type, maltreatment type, and short-term child protection service outcome. More than a third of Asian-Canadian children and families in the CIS-2008 were investigated (35.5\%) and substantiated (36.6\%) for physical abuse, compared to White-Canadian children and families. Substantiated child maltreatment investigations for Asian-Canadian children and families was less than half that of White-Canadian children and families to be transferred to ongoing child protection service (19.3\% vs. $43.1 \%)$. The differences in case characteristics and service provision are consistent with this author's previous study (e.g., Author, 2014). The results from focus groups with child welfare workers validate the results from secondary data analysis using the CIS-2008, and offer a cultural and structural context for understanding child welfare involvement with Asian-Canadians. Information regarding the immigration status, level of acculturation and affiliation is not available and further research is needed in this area. 


\section{References}

Author. (2014). Physical abuse and Asian families in the Canadian child welfare system. Journal of Aggression, Maltreatment and Trauma, 23(5), 532-551. doi:

10.1080/10926771.2014.904462

Ards, S., Chung, C., \& Myers, S. L. (1998). The effects of sample selection bias on racial differences in child abuse reporting. Child Abuse and Neglect, 22(2), 103-115. doi: 10.1016/S0145-2134(97)00131-2

Baird, C., Wagner, D., Healy, T., \& Johnson, K. (1999). Risk assessment in child protective services: Consensus and actuarial model reliability. Child Welfare, 78(6), 723-748.

Barnett, L. (2008). The “spanking” law: Section 43 of the Criminal Code. Ottawa, ON: Library of Parliament.

Behl, L. E., Crouch, J. L., May, P. F., Valente, A. L., \& Conyngham, H. A. (2001). Ethnicity in child maltreatment research: A content analysis. Child Welfare, 6(2), 143-147. doi: $10.1177 / 1077559501006002006$

Bland, M. J. (1995). Multiple significance tests: the Bonferroni method. British Medical Journal, 310, 170. doi: http://dx.doi.org/10.1136/bmj.310.6973.170

Boeije, H. (2002). A purposeful approach to the constant comparative method in analyzing qualitative interviews. Quality and Quantity, 36(4), 391-409. doi:

10.1023/A:1020909529486

Boytzis, R. E. (1998). Transforming Qualitative Information: Thematic Analysis and Code Development. Thousand Oaks: Sage Publications.

Breton, R. (2005). Immigrant ethnic groups and social incorporation. In Ethnic relations in Canada: Institutional dynamics (pp. 184-201). Montreal: McGill-Queen's University Press.

Brod, M., Tesler, L.E., \& Christensen, T.L. (2009) Qualitative research and content validity: Developing best practices based on science and experience. Quality of Life Research, 18(9), 1263-78.

Canadian Foundation for Children, Youth and the Law v. Canada (Attorney General), 2004 SCC 4, aff'g (2002), 57 O. R. (3d) 511 (C. A.), aff'g (2000), 49 O. R. (3d) 662 (S. C.) [“CFCYL v. Canada"].

Chan, Y. C., Lam, G. L. T., \& Shae, W. C. (2011). Children's views on child abuse and neglect: Findings from an exploratory study with Chinese children in Hong Kong. Child Abuse and Neglect, 35(3), 162-72. doi: 10.1016/j.chiabu.2010.12.001

Chao, R. K. (1994). Beyond parental control and authoritarian parenting style: Understanding 
Chinese parenting through the cultural notion of training. Child Development, 65, 11111119. doi: 10.1111/j.1467-8624.1994.tb00806.x

Child and Family Services Act, R.S.O. 1990, c. C.11.

Chibnall, S., Dutch, N. M., Jones-Harden, B., Brown, A., Gourdine, R., Smith, J., Boone, A., \& Snyder, S. (2003). Children of colour in the child welfare system: Perspectives from the child welfare community. U.S. Department of Health and Human Services, Administration for Children and Families and Children's Bureau.

Clarke, J. (2011). The challenges of child welfare involvement for Afro-Caribbean families in Toronto. Children and Youth Services Review, 33(2011), 274-283. doi: 10.1016/j.childyouth.2010.09.010

Creswell, J. W., \& Clark, V. L. P. (2011). Designing and Conducting Mixed Methods Research $\left(2^{\text {nd }} e d.\right)$. Thousand Oaks, CA: Sage Publications.

Criminal Code, R.S.C. 1985, c. C-46.

Dakil, S. R., Cox, M., Lin, H., \& Flores, G. (2011). Racial and ethnic disparities in physical abuse reporting and child protective services interventions in the United States. Journal of National Medical Association, 103(9-10), 926-31.

Durrant, J., Trocmé, N., Fallon, B., Milne, C., \& Black, T. (2009). Protection of children from physical maltreatment in Canada: An evaluation of the Supreme Court's definition of reasonable force. Journal of Aggression, Maltreatment \& Trauma, 18, 64-87. doi: $10.1080 / 10926770802610640$

Elliot, R., \& Timulak, L. (2005). Descriptive and interpretive approaches to qualitative research. In J. Miles \& Gilbert (eds.), A Handbook of Research Methods in Clinical and Health Psychology (147-159). Oxford, UK: Oxford University Press.

Fallon, B., MacLaurin, B., Trocmé, N., \& Felstiner, C. (2003). A national profile of child protection workers. In K. Kufeldt and B. MacKenzie (Eds.), Child welfare: Connecting research, policy and practice (pp. 41-52). Waterloo, ON: Wilfred Laurier University Press.

Fluke, J., Harden, B. J., Jenkins, M., \& Ruehrdanz, A. (2010). Research synthesis on child welfare disproportionality and disparities. American Humane Association. Retrieved from http://www.cssp.org/publications/child-welfare/alliance/Disparities-and-Disproportionalityin-Child-Welfare_An-Analysis-of-the-Research-December-2011.pdf\#page =11

Garland, A. F., Landsverk, J. A., \& Lau, A. S. (2003). Racial/ethnic disparities in mental health service use among children in foster care. Children and Youth Services Review, 25(5/6), 491-507. doi: 10.1016/S0190-7409(03)00032-X

Hennink, M. M. (2007). International Focus Group Research: A Handbook for the Health and 
Social Sciences. Cambridge: Cambridge University Press.

Hill, R. B. (2006). Synthesis of research on disproportionality in child welfare: An update. The Casey/Center for the Study of Social Policy Alliance for Racial Equity. Retrieved from http://www.caseyfamilyservices.org/resources/pubdetail/266/

Hill, R. B. (2007). An analysis of racial/ethnic disproportionality and disparity at the national, state, and county levels. Casey- CSSP Alliance for Racial Equity in Child Welfare.

Hines, A. M., Lemon, K., Wyatt, P., \& Merdinger, J. (2004). Factors related to the disproportionate involvement of children of color in the child welfare system: A review and emerging themes. Children and Youth Services Review, 26(2004), 507-527. doi: 10.1016/j.childyouth.2004.01.007

Ho, J., Yeh, M., McCabe, K., \& Lau, A. (2012). Perceptions of the acceptability of parental training among Chinese immigrant parents: Contributions of cultural factors and clinical needs. Behavior Therapy, 43(2012), 436-449. doi: 10.1016/j.beth.2011.10.004

Huang, K. Y., Calzada, E. J., Cheng, S., Brotman, L. M. (2012). Physical and mental health disparities among young children of Asian immigrants. Journal of Pediatrics, 160, 331336. doi:10.1016/j.jpeds.2011.08.005

Jent, J. F., Eaton, C. K., Knickerbocker, L., Lambert, W. F., Merrick, M. T., \& Dandes, S. K. (2011). Multidisciplinary child protection decision-making about physical abuse: Determining substantiation thresholds and biases. Children and Youth Service Review, 33(2011), 1673-1682. doi:10.1016/j.childyouth.2011.04.029

Ju, S., \& Lee, Y. (2010). Experiences of family maltreatment by Korean children in Korean National Protective Services. Child Abuse and Neglect, 34(2010), 18-27. doi: 10.1016/j.chiabu.2009.09.009

Kim, E., Cain, K. C., \& Webster-Stratton, C. (2008). The preliminary effect of a parenting program for Korean American mothers: A randomized controlled experimental study. International Journal of Nursing Studies, 45, 1261-1273.

Kim, W., \& Keefe, R. H. (2010). Barriers to healthcare among Asian Americans. Social Work in Public Health, 25, 286-295. doi: 10.1080/19371910903240704

Kriz, K., \& Skivenes, M. (2012). Child-centric or family focused? A study of child welfare workers' perceptions of ethnic minority children in England and Norway. Child and Family Social Work, 17, 448-457. doi: 10.1111/j.1365-2206.2011.00802.x

Lau, A. S. (2010). Physical discipline in Chinese American immigrant families: An adaptive culture perspective. Cultural Diversity and Ethnic Minority Psychology, 1(3), 313-322. doi: $10.1037 / \mathrm{a} 0018667$ 
Lau, J. T. F., Chan, K. K., Lam, P. K. W., Choi, P. Y. W., Lai, K. Y. C. (2003). Psychological correlates of physical maltreatment in Hong Kong Chinese adolescents. Child Abuse and Neglect, 27, 63-75. doi: 10.1016/S0145-2134(02)00507-0.

Lau, A. S., Fung, J. J., Ho, L. Y., Liu, L., \& Gudiño, O. G. (2011). Parent training with high-risk immigrant Chinese families: A pilot trial yielding practice-based evidence. Behavior Therapy, 42, 413-426.

Lee, S. J., Soeck, J. L., Djelaj, V., \& Agius, E. (2013). When practice and policy collide: Child welfare workers' perceptions of investigation processes. Children and Youth Service Review, 35(2013), 634-641. doi: 10.1016/j.childyouth.2013.01.004

Leong, F. T. L., \& Lau, A. S. L. (2001). Barriers to providing effective mental health services to Asian Americans. Mental Health Services Research, 3(4), 201-214. doi: 10.1023/A:1013177014788

Leung, C., Tsang, S., Heung, K., \& Yiu, I. (2009). Effectiveness of parent-child interaction therapy (PCIT) among Chinese families. Research on Social Work Practice, 19, 304-313. doi: $10.1177 / 1049731513519827$

Lieber, E., Fung, H., \& Leung, P. W. L. (2006). Chinese child-rearing beliefs: Key dimensions and contributions to the development of culture-appropriate assessment. Asian Journal of Social Psychology, 9, 140-147. doi: 10.1111/j.1467-839X.2006.00191.x

Ontario Association of Children's Aid Society (OACAS). (2006). Ontario Child Welfare Eligibility Spectrum. Toronto, ON: OACAS.

Park, M. S. (2001). The factors of child physical abuse in Korean immigrant families. Child Abuse and Neglect, 25(2001), 945-958. doi:10.1016/S0145-2134(01)00248-4

Rhind, N., Leung, T., \& Choi, F. (1999). Child sexual abuse in Hong Kong: Double victimization? Child Abuse and Neglect, 23(5), 511-517. doi:10.1016/S01452134(99)00016-2

Rivaux, S. L., James, J., Wittenstrom, K., Daunaunn, D., Sheets, J, Henry, J., \& Jeffries, V. (2008). The intersection of race, poverty, and risk: Understanding the decisions to provide services to clients and to remove children. Child Welfare, 87(2), 151-168.

Saldana, J. (2013). The coding manual for qualitative researchers. (2 ${ }^{\text {nd }}$ Ed.). Los Angeles, CA: SAGE.

Scheppers, E., van Donge, E., Dekker, J., Geertzen, J., \& Dekker, J. (2006). Potential barriers to the use of health services among ethnic minorities: A review. Family Practice - An International Journal, 23, 325-348. doi: 10.1093/fampra/cmi113 
Sinha, V., Trocmé, N., Fallon, B., MacLaurin, B., Fast, E., \& Thomas Prokop, S., et al (2011). Kiskisik Awasisak: Remember the Children. Understanding the Overrepresentation of First Nations Children in the Child Welfare System. Ontario: Assembly of First Nations.

Statistics Canada. (2010). Projections of the Diversity of the Canadian Population, 2006 to 2031. Catalogue no. 91-551-X. Retrieved from: http://www.statcan.gc.ca/pub/91-551-x/91-551x2010001-eng.pdf

Tang, C. S. K. (2006). Corporal punishment and physical maltreatment against children: A community study on Chinese parents in Hong Kong. Child Abuse and Neglect, 30, 893-907. doi: 10.1016/j.chiabu.2006.02.012.

Tang, C. S. K., Wong, W. C., Leung, P. M., Chen, W. Q., Lee, A., \& Ling, D. C. (2011). Health compromising behaviors among Chinese adolescents: Role of physical abuse, school experience, and social support. Journal of Health Psychology, 16(3), 457-466. doi: $10.1177 / 1359105310384297$

Terao, S. Y., Borrego, J. Jr., \& Urquiza, A. J. (2001). A reporting and response model for culture and child maltreatment. Child Maltreatment, 6(2), 158-168. doi:10.1177/1359105310384297

Trocmé, N., Fallon, B., MacLaurin, B., Sinha, V., Black, T., et al. (2010). Methodology. In Canadian Incidence Study of Reported Child Abuse and Neglect-2008: Major Findings. PHAC: Ottawa.

U.S. Census Bureau. (2012). The Asian population: 2010. U.S. Census Bureau. Retrieved from: http://www.census.gov/prod/cen2010/briefs/c2010br-11.pdf

UNICEF. (2005). Regional assessment on violence against children in East Asia and the Pacific, desk review 2005. UNICEF: Bangkok, Thailand.

Zhai, F. \& Gao, Q. (2009). Child maltreatment among Asian Americans: Characteristics and explanatory framework. Child Maltreatment, 14(2), 207-224. doi: $10.1177 / 1077559508326286$

Zhu, Y., \& Tang, K. L. (2012). Physical child abuses in urban China: Victims' perceptions of the problem and impediments to help-seeking. International Social Work, 55(4), 574-588. doi: $10.1177 / 0020872811425806$ 
Table 1. Participant Demographic, Education, and Practice Experience $(\mathrm{N}=18)$

\begin{tabular}{|c|c|}
\hline Demographics & $\mathbf{n}$ \\
\hline \multicolumn{2}{|l|}{ Worker Gender } \\
\hline Male & 3 \\
\hline Female & 15 \\
\hline \multicolumn{2}{|l|}{ Worker Ethnicity } \\
\hline Asian & 10 \\
\hline Black & 4 \\
\hline Latino/Hispanic & 2 \\
\hline White/Caucasian & 1 \\
\hline Multi-Racial & 1 \\
\hline \multicolumn{2}{|l|}{ Highest Social Work Degree } \\
\hline Bachelor & 7 \\
\hline Master/PhD & 11 \\
\hline \multicolumn{2}{|c|}{ Non-Child Protection Experience - Years } \\
\hline$<1$ & 10 \\
\hline 1 to 4 & 4 \\
\hline$>5$ & 4 \\
\hline \multicolumn{2}{|c|}{ Direct Child Protection Experience } \\
\hline Intake Screening/Investigation ${ }^{a}$ & 0 \\
\hline Ongoing Family Services ${ }^{b}$ & 3 \\
\hline Children's Services ${ }^{\mathrm{c}}$ & 3 \\
\hline Multiple Positions $^{\mathrm{d}}$ & 10 \\
\hline Non-Protection ${ }^{\mathrm{e}}$ & 3 \\
\hline \multicolumn{2}{|c|}{ Child Protection Experience - Years } \\
\hline$<1$ & 3 \\
\hline 1 to 4 & 3 \\
\hline 5 to 9 & 2 \\
\hline 10 to 14 & 7 \\
\hline 15 to 19 & 0 \\
\hline$>20$ & 3 \\
\hline
\end{tabular}

${ }^{\text {a }}$ Intake screening/Investigation is the initial receipt and investigation on allegations of child maltreatment.

${ }^{\mathrm{b}}$ Ongoing family service is the continuation of child protection services.

${ }^{\mathrm{c}}$ Children's service is specific support and services for children/youth in out-of-home care.

${ }^{\mathrm{d}}$ Multiple positions are for those who have worked in more than one category of intake screening/investigation, ongoing family services, or children's services.

${ }^{\mathrm{e}}$ Non-protection includes community social service professionals working with children and their families outside of the child protection system. 
Table 2. Demographic Characteristics of Maltreatment-related Investigations by Asian-Canadian and White-Canadian Children and Families in Canada in $2008(\mathrm{~N}=159,325)$

\begin{tabular}{|c|c|c|c|c|c|}
\hline \multirow[b]{2}{*}{ Demographics } & \multicolumn{2}{|c|}{ Asian-Canadian } & \multicolumn{2}{|c|}{ White-Canadian } & \multirow[b]{2}{*}{$\chi^{2}$} \\
\hline & Estimate & $\%$ & Estimate & $\%$ & \\
\hline $\begin{array}{l}\text { Total Child Maltreatment- } \\
\text { related Investigation }\end{array}$ & 5,988 & 100 & 153,337 & 100 & \\
\hline Gender of Child & & & & & 0.66 \\
\hline Male & 3,170 & 52.9 & 77,650 & 52.9 & \\
\hline Female & 2,818 & 47.1 & 75,687 & 47.1 & \\
\hline Child Age - Years & & & & & 0.48 \\
\hline 4 years and Under & 1,734 & 29.0 & 46,882 & 30.6 & \\
\hline 5 years and Over & 4,254 & 71.0 & 106,455 & 69.4 & \\
\hline $\begin{array}{l}\text { Number of Children in the } \\
\text { Family }\end{array}$ & & & & & 0.659 \\
\hline One or Two Children & 3,733 & 62.3 & 98,559 & 64.3 & \\
\hline More than Two Children & 2,255 & 37.7 & 54,778 & 35.7 & \\
\hline Household Composition & & & & & $74.35 * * *$ \\
\hline Single Caregiver & 1,095 & 18.7 & 62,521 & 40.8 & \\
\hline Two Caregivers & 4,751 & 81.3 & 90,816 & 59.2 & \\
\hline Two Caregiver Relationship & & & & & $113.14 * * *$ \\
\hline Biological Parent & 4,266 & 93.8 & 53,224 & 63.5 & \\
\hline All Other Relationships & 282 & 6.2 & 30,537 & 36.5 & \\
\hline Household Source of Income & & & & & $74.82 * * *$ \\
\hline Full-time & 4,395 & 73.4 & 86,383 & 56.3 & \\
\hline Part-time/Seasonal & 357 & 6.0 & 15,407 & 10.0 & \\
\hline Other Benefits or Unemployed & 632 & 10.6 & 41,908 & 27.3 & \\
\hline Unknown Source & 434 & 7.2 & 7,524 & 4.9 & \\
\hline No Source of Income & - & - & 2,115 & 1.4 & \\
\hline
\end{tabular}

Note. Estimate based on an unweighted sample of 10,770 child maltreatment-related investigations for children 15 years and under, involving 380 Asian-Canadian and 10,390 White-Canadian.

- Represents weighted estimates $<100$. These estimates were too small to report according to CIS regulations.

$* * * p<.001$. 
Table 1. Child Maltreatment-related Investigations involving Asian-Canadian and WhiteCanadian Children and Families in Canada in $2008(\mathrm{~N}=159,325)$

\begin{tabular}{|c|c|c|c|c|c|}
\hline \multirow[b]{2}{*}{ Variables } & \multicolumn{2}{|c|}{ Asian-Canadian } & \multicolumn{2}{|c|}{ White-Canadian } & \multirow[b]{2}{*}{$\chi^{2}$} \\
\hline & Estimate & $\%$ & Estimate & $\%$ & \\
\hline $\begin{array}{l}\text { Total Child Maltreatment- } \\
\text { related Investigation }\end{array}$ & 5,988 & 100 & 153,337 & 100 & \\
\hline Investigation Type & & & & & $21.88 * * *$ \\
\hline Risk Investigation Only & 911 & 15.2 & 39,357 & 25.7 & \\
\hline Maltreatment Investigation & 5,077 & 84.8 & 113,980 & 74.3 & \\
\hline Primary Maltreatment Type & & & & & $84.02 * * *$ \\
\hline Physical Abuse & 2,124 & 35.5 & 29,002 & 18.9 & \\
\hline Sexual Abuse & - & - & 7,940 & 5.2 & \\
\hline Neglect & 1,384 & 23.1 & 39,496 & 25.8 & \\
\hline Emotional Maltreatment & 368 & 6.1 & 11,353 & 7.4 & \\
\hline Exposure Domestic Violence & 1,124 & 18.8 & 26,189 & 17.1 & \\
\hline Substantiation Decision & & & & & 10.69 \\
\hline Unfounded & 1,773 & 34.9 & 48,804 & 42.8 & \\
\hline Suspected & 484 & 9.5 & 12,194 & 10.7 & \\
\hline Substantiated & 2,821 & 55.6 & 52,982 & 46.5 & \\
\hline
\end{tabular}

Note. Estimate based on an unweighted sample of 10,770 child maltreatment-related investigations for children 15 years and under, involving 380 Asian-Canadian and 10,390 White-Canadian.

- Represents weighted estimates $<100$. These estimates were too small to report according to CIS regulations.

${ }_{* * *} p<.001$.

Table 2. Substantiated Child Maltreatment Investigations by Asian-Canadian and WhiteCanadian Households in Canada in $2008(\mathrm{~N}=55,802)$

\begin{tabular}{|c|c|c|c|c|c|}
\hline \multirow[b]{2}{*}{ Variables } & \multicolumn{2}{|c|}{ Asian-Canadian } & \multicolumn{2}{|c|}{ White-Canadian } & \multirow[b]{2}{*}{$\chi^{2}$} \\
\hline & Estimate & $\%$ & Estimate & $\%$ & \\
\hline $\begin{array}{l}\text { Total Substantiated Child } \\
\text { Maltreatment Investigation }\end{array}$ & 2,820 & 100 & 52,982 & 100 & \\
\hline Referral Source & & & & & $20.92 * * *$ \\
\hline Professionals & 2,544 & 90.2 & 40,057 & 75.6 & \\
\hline Non-professionals & 277 & 9.8 & 12,925 & 24.4 & \\
\hline Primary Maltreatment Type & & & & & $29.35 * * *$ \\
\hline Physical Abuse & 1,033 & 36.6 & 11,204 & 21.1 & \\
\hline Sexual Abuse & - & - & 1,964 & 3.7 & \\
\hline Neglect & 638 & 22.6 & 16,567 & 31.3 & \\
\hline Emotional Maltreatment & 248 & 8.8 & 5,248 & 9.9 & \\
\hline Exposure Domestic Violence & 880 & 31.2 & 17,999 & 34.0 & \\
\hline \multicolumn{6}{|l|}{ Transfer to Ongoing } \\
\hline Yes & 544 & 19.3 & 22,800 & 43.1 & $40.29 * * *$ \\
\hline No & 2,276 & 80.7 & 30,182 & 56.9 & \\
\hline
\end{tabular}

